Pacific

Journal of

Mathematics

UNIQUE FUNCTIONALS AND REPRESENTATIONS

OF HECKE ALGEBRAS

BENJAMIN BRUBAKER, DANIEL BUMP AND SOLOMON FRIEDBERG 


\title{
UNIQUE FUNCTIONALS AND REPRESENTATIONS OF HECKE ALGEBRAS
}

\author{
BEnJAmin BRUBAKER, DANIEl Bump AND SOLOMON FriedBERG
}

To the memory of Jonathan David Rogawski

\begin{abstract}
Rogawski (1985) used the affine Hecke algebra to model the intertwining operators of unramified principal series representations of $\boldsymbol{p}$-adic groups. On the other hand, a representation of this Hecke algebra in which the standard generators act by Demazure-Lusztig operators was introduced by Lusztig (1989) and applied by Kazhdan and Lusztig (1987) to prove the Deligne-Langlands conjecture. These operators appear in various other contexts. Ion (2006) used them to express matrix coefficients of principal series representations in terms of nonsymmetric Macdonald polynomials, while Brubaker, Bump and Licata (2011) found essentially the same operators underlying recursive relationships for Whittaker functions. Here we explain the role of unique functionals and Hecke algebras in these contexts and revisit the results of Ion from the point of view of Brubaker et al.
\end{abstract}

\section{Introduction}

One of the innovations in [Rogawski 1985] was the use of the Hecke algebra to model the intertwining operators of unramified principal series representations of $p$-adic groups. His goal was the classification of the irreducible representations of the Hecke algebra, or equivalently, the irreducible representations of a $p$-adic group having an Iwahori-fixed vector. These had already been classified in the case of $\mathrm{GL}_{n}$ by Zelevinsky [1980]. It was known from [Zelevinsky 1981] that there were analogies between this problem and the decomposition of Verma modules of a semisimple Lie algebra into irreducibles, where deep connections with the theory of Hecke algebras had been found by Kazhdan and Lusztig [1979]. Rogawski sought to clarify the relationship between $p$-adic representation theory and Kazhdan-Lusztig

This work was partially supported by NSF grants DMS-0844185, DMS-1001079 and DMS-1001326, and by NSA grant H98230-10-1-0183.

MSC2010: primary 22E50; secondary 22E35, 33D52.

Keywords: Hecke algebra, unramified principal series, Demazure-Lusztig operator, unique functional. 
theory. As part of this effort, he expressed intertwining integrals between principal series representations in terms of Kazhdan-Lusztig elements of the Hecke algebra.

Among the tools that had been brought to bear on the study of Verma modules, Jantzen [1979] had introduced a filtration of the Verma module based on the Shapovalov bilinear form. With the analogy between $p$-adic groups and Verma modules in mind, Rogawski gave an analog of the Jantzen filtration, and also reproved the results of Zelevinsky [1980] for $\mathrm{GL}_{n}$ using Hecke algebra methods.

After [Rogawski 1985] was written, Zelevinsky's results were generalized to arbitrary $p$-adic groups by Kazhdan and Lusztig [1985; 1987], who proved the Langlands-Deligne conjecture classifying the irreducible representations of a $p$-adic group $G$ that have Iwahori-fixed vectors. They made use of a representation of the affine Hecke algebra $\mathscr{H}_{J}$ on the ring of rational functions on the maximal torus $\hat{T}$ of the L-group $\hat{G}$. In this action, the generators of the Hecke algebra act by certain operators known as Demazure-Lusztig operators, which resemble the well-known Demazure operators that occur in the cohomology of line bundles over Schubert varieties. Given data consisting of a pair $(s, u)$ of elements of $\hat{G}$ such that $s$ is semisimple, $u$ is unipotent, and $s u s^{-1}=u^{q}$, where $q$ is the cardinality of the residue field, a subquotient of this representation can be found that gives an irreducible $\mathscr{H}_{J}$-module. This module may be identified with the space of Iwahori-fixed vectors in an irreducible representation of $G(F)$. To prove that this gives every irreducible representation of $G(F)$ uniquely, thus proving the Deligne-Lusztig conjecture, Kazhdan and Lusztig made use of the "coincidence" that the same representation of the Hecke algebra by Demazure-Lusztig operators also occurs in the K-theory of flag varieties, allowing statements about representations to be translated into algebraic geometry, where suitable methods are available.

The representation of the Hecke algebra by Demazure-Lusztig operators comes up in yet another context, namely the study of special functions on a $p$-adic group realized as matrix coefficients involving Iwahori-fixed vectors. Our first task, after some preliminaries, will be to briefly retrace Rogawski's steps and to discuss the relationship between the Hecke algebra and the intertwining operators. The intertwining operators involve different principal series representations, which must be taken together to obtain a representation of the Hecke algebra. We express this by saying that the principal series representation is variable in this representation of the Hecke algebra.

As we will explain in Section 3, this representation may be converted into something concrete by introducing a family of functionals on the principal series representations. We will consider two particular such families: the Whittaker functionals and the spherical functionals. The role of the unique functional is that it converts the Iwahori-fixed vectors in a variable principal series representation into a family of regular functions on $\hat{T}$. The problem of variability of the principal 
series representation disappears, and the action of the Hecke algebra generators is by some variant (depending on the functional) of the Demazure-Lusztig operator. The regularity of the functions obtained this way is related to Bernstein's method of showing that a unique functional defined (typically by a suitable integral) on an open subspace extends to a meromorphic function for all Langlands parameters. (See, for example, [Banks 1998] or [Sakellaridis 2006, Section 7] for Bernstein's method.)

Both the spherical and the Whittaker functionals are defined and nonzero on a Zariski dense set of $\hat{T}$. By contrast, one may consider functionals defined and nonzero on only a subset of $\hat{T}$ that is not Zariski dense. An example is the Shalika functional on representations of $\mathrm{GL}_{n}$, proved unique by Jacquet and Rallis [1996], with a Casselman-Shalika type formula established by Sakellaridis [2006]. The Shalika functional, like the Bessel models for classical groups, exhibits characteristics of both the spherical and Whittaker functionals and can be studied using our methods. We hope to return to this in a later paper.

Shortly before [Rogawski 1985] appeared, intertwining operators had been used for another purpose: the computation of the spherical vector in each of our two archetypal models. For the spherical model, Casselman [1980] used intertwining operators to give a new proof of the Macdonald formula which expresses the values of the spherical function as specializations of Macdonald symmetric functions. This specialization is the Hall-Littlewood polynomial in the case of $\mathrm{GL}_{n}$. Later, nonsymmetric Macdonald polynomials were defined in [Macdonald 2003; Opdam 1995; Cherednik 1995]. Generalizing the Macdonald formula, Ion [2006] showed that the Iwahori-fixed vectors in the model are expressible in terms of nonsymmetric Macdonald polynomials, making use of recursions that they satisfy involving Demazure-Lusztig operators. See also [Cherednik and Ostrik 2003] for earlier hints of this connection.

For the Whittaker functional, Casselman and Shalika [1980] used the intertwining operators to show that the values of the spherical Whittaker function are the irreducible characters of $\hat{G}$, multiplied by a factor which is a deformation of the denominator in the Weyl character formula. Regarding the more general Iwahorifixed vectors in the model, Reeder [1992; 1993] used the Hecke algebra action to give recursions between these, usable for explicit computation. These relations can be understood in terms of Demazure-Lusztig operators as proved by Brubaker, Bump and Licata [Brubaker, Bump and Licata 2011].

In Section 2, we review the relation between the Iwahori Hecke algebra and the intertwining operators for principal series. Then, in Section 3, we show that, given a unique functional on a Zariski dense subset of $\hat{T}$, to each generator $T_{i}$ of the Iwahori Hecke algebra we may attach a difference operator on a suitable ring of regular functions that is similar to a Demazure operator. This extends to an 
action of the Hecke algebra. In the case of the spherical functional, this gives a new perspective on the work of Ion [2006].

\section{Hecke algebras and intertwiners of principal series}

Let $G$ be a reductive algebraic group defined and split over a nonarchimedean local field $F$ with ring $\mathfrak{o}$ of integers and prime $\mathfrak{p}$. We may regard $G$ as defined over $\mathfrak{o}$ in such a way that $K=G(\mathfrak{o})$ is a special maximal compact subgroup. Let $q=|\mathfrak{o} / \mathfrak{p}|$. Let $T$ be a split maximal torus contained in Borel subgroup $B$, and let $W_{0}=N(T) / T$ be the Weyl group. We will always choose representatives for $W_{0}$ from $N(T) \cap K$.

The connected L-group $\hat{G}$ is an algebraic group defined over $\mathbb{C}$ with a maximal torus $\hat{T}$ that is in duality with $T$ in the sense that elements of $\hat{T}(\mathbb{C})$ are in bijection with the unramified characters of $T(F)$, that is, those that are trivial on $T(F) \cap K=$ $T(\mathfrak{o})$. Let $J$ be the Iwahori subgroup, which is the preimage in $K$ of $B\left(\mathbb{F}_{q}\right)$ under the canonical homomorphism $G(\mathfrak{o}) \rightarrow G\left(\mathbb{F}_{q}\right)$.

Let $s_{1}, \ldots, s_{r}$ be the simple reflections in the Weyl group $W_{0}$. The affine Weyl group $W_{\text {aff }}$ is obtained by adjoining one more "affine" simple reflection $s_{0}$ [Bourbaki 1968, Section VI.2]. It is the semidirect product of $W_{0}$ by the root lattice $Q^{\vee}$ of $\hat{T}$, which is the coroot lattice of $T$. The groups $W_{0}$ and $W_{\text {aff }}$ are Coxeter groups. The extended affine Weyl group $W_{\text {ext }}$ is a slightly larger group that is the semidirect product of $W_{0}$ by the weight lattice $P^{\vee}$ of $\hat{T}$, which is the coweight lattice of $T$, isomorphic to $T(F) / T(\mathfrak{o})$. In this presentation, $P^{\vee}$ is a normal subgroup. We will denote by $\lambda \mapsto a_{\lambda}$ a map sending $\lambda \in P^{\vee}$ to a representative of the corresponding coset in $T(F) / T(\mathfrak{o})$.

The group $W_{\text {ext }}$ is not a Coxeter group, but like Coxeter groups, it has a length function. The finite subgroup $\Omega$ of elements of length 0 is isomorphic to $P^{\vee} / Q^{\vee}$. For example, if $G$ is semisimple, then $\Omega$ is isomorphic to the fundamental group of $G$. The group $W_{\text {ext }}$ is the semidirect product of $W_{\text {aff }}$ by $\Omega$, with $W_{\text {aff }}$ being a normal subgroup. Conjugation by an element of $\Omega$ permutes the $s_{i}$.

The (affine) Iwahori Hecke algebra $\mathscr{H}_{J}$ is the convolution algebra of compactly supported $J$-biinvariant functions on $G(F)$. Let $\mathscr{H}_{0}$ be the subring of functions with support in $K$. Then $\mathscr{H}_{0}$ and $\mathscr{H}_{J}$ have the following explicit description due to Iwahori and Matsumoto [1965]. If $r$ is the rank of $G$, the ring $\mathscr{H}_{0}$ is generated by $T_{1}, \ldots, T_{r}$, where each $T_{i}$ is the characteristic function of $J s_{i} J$. These $T_{i}$ then satisfy quadratic relations

$$
T_{i}^{2}=(q-1) T_{i}+q
$$

and the braid relations

$$
T_{i} T_{j} T_{i} \ldots=T_{j} T_{i} T_{j} \ldots,
$$


where the number of terms on each side is the order of $s_{i} s_{j}$. The affine Hecke algebra $\mathscr{H}_{\text {aff }}$ is obtained by adjoining an element $T_{0}$ satisfying the same quadratic and braid relations but allowing $i=0$ corresponding to the affine simple reflection $s_{0}$. The algebra $\mathscr{H}_{J}$ is slightly larger than $\mathscr{H}_{\text {aff }}$, and is isomorphic as a vector space to $\mathscr{H}_{\text {aff }} \otimes \mathbb{C}[\Omega]$. The elements of $\Omega$ act on $\mathscr{H}_{\text {aff }}$ by conjugation, and this action corresponds to permuting the $T_{i}$, just as in $W_{\text {ext }}$ conjugation by elements of $\Omega$ permutes the $s_{i}$.

The algebra $\mathscr{H}_{J}$ also has a presentation analogous to the presentation of $W_{\mathrm{ext}}$ as the semidirect product of $P^{\vee}$ by $W_{0}$. This presentation, sometimes known as the Bernstein presentation, was developed but not published by Bernstein and Zelevinsky. Possibly its first use in a published paper was in [Rogawski 1985], and a full treatment was given by Lusztig [1989]. In the Bernstein presentation, $\mathscr{H}_{0}$ is supplemented by a ring homomorphism $\theta: \mathbb{C}\left[P^{\vee}\right] \rightarrow \mathscr{H}_{J}$. As a vector space, $\mathscr{H}_{J}=\mathscr{H}_{0} \otimes \mathbb{C}\left[P^{\vee}\right]$. To describe the ring structure, it is sufficient to give one relation. Let $1 \leqslant i \leqslant r$ and let $\lambda \in P^{\vee}$. Then

$$
\theta(\lambda) T_{i}-T_{i} \theta\left(s_{i} \lambda\right)=(q-1) \frac{\theta(\lambda)-\theta\left(s_{i} \lambda\right)}{1-\theta\left(-\alpha_{i}^{\vee}\right)},
$$

where $\alpha_{i}^{\vee}$ is the coroot corresponding to $i$.

Let $z \in \hat{T}(\mathbb{C})$, and let $\tau=\tau_{z}: T(F) \rightarrow \mathbb{C}^{\times}$be the corresponding unramified character. We extend it to $B(F)$ by letting the unipotent radical $N(F)$ be in the kernel. The principal series representation $M(\tau)$ consists of all locally constant maps $f: G(F) \rightarrow \mathbb{C}$ such that $f(b g)=\left(\delta^{1 / 2} \tau\right)(b) f(g)$ for $b \in B(F), g \in G(F)$. The action of $G(F)$ is by right translation. The module $M(\tau)$ is irreducible if $\tau$ is in general position.

If $(\pi, V)$ is an irreducible representation having a $J$-fixed vector, then $V^{J}$ is a finite-dimensional irreducible $\mathscr{H}_{J}$ module and its isomorphism class determines $\pi$. Any such $(\pi, V)$ with a $J$-fixed vector is a subquotient of $M(\tau)$ for some $\tau$, and the category of smooth representations of finite length all of whose composition factors have $J$-fixed vectors is equivalent to the category of finite-dimensional $\mathscr{H}_{J}$-modules.

The Weyl group $W_{0}$ acts on $T$ and hence on unramified characters. We will make this a right action, so $\tau w(a)=\tau\left(w a w^{-1}\right)$ for $a \in T(F)$. If $w \in W_{0}$, the modules $M(\tau)$ and $M(\tau w)$ are isomorphic if irreducible, and in any case have isomorphic semisimplifications. To see this, one may construct homomorphisms $A_{w}: M(\tau) \rightarrow M(\tau w)$ by means of intertwining integrals. By definition,

$$
A_{w} f(g)=\int_{N \cap w^{-1} N_{-} w} f(w n g) d n
$$


where $N_{-}$is the unipotent radical of the negative Borel. The integral is convergent if $\left|\tau\left(a_{\lambda}\right)\right|<1$ for dominant weights $\lambda$. By the singular set, we mean the union of hyperplanes in $\hat{T}(\mathbb{C})$ that are the kernels of the coroots $\alpha^{\vee}$ (regarded as characters of $\hat{T}$ ). For arbitrary $\tau$, the intertwining operator may be defined by analytic continuation, except that the $A_{w}$ can have poles in the singular set.

Now we return to the point in question: why may Hecke algebras be used to model intertwining operators? The basic insight is that, to any ring $R$ regarded as a left $R$-module, a left $R$-module homomorphism $\lambda: R \rightarrow R$ is necessarily of the form $\lambda(x)=x \cdot a$ for some $a \in R$. This is trivial to prove with $a=\lambda(1)$.

The first way of applying this is to note that the space $M(\tau)^{J}$ of $J$-invariants is $\left|W_{0}\right|$-dimensional. It has several natural bases indexed by Weyl group elements. A particular one is the basis $\Phi_{w}^{\tau}=\Phi_{w}\left(w \in W_{0}\right)$ defined by

$$
\Phi_{w}^{\tau}(b k)= \begin{cases}\delta^{1 / 2} \tau(b) & \text { if } k \in B w J \\ 0 & \text { otherwise }\end{cases}
$$

for $b \in B(F)$ and $k \in K$.

We see that $\mathscr{H}_{0}$ and $M(\tau)^{J}$ are both $\left|W_{0}\right|$-dimensional $\mathscr{H}_{0}$-modules, and in fact they are isomorphic as left $\mathscr{H}_{0}$-modules. A particular isomorphism

$$
\varrho_{\tau}: M(\tau)^{J} \rightarrow \mathscr{H}_{0}
$$

is given by $\varrho_{\tau}(f)=F$, where

$$
F(g)= \begin{cases}f\left(g^{-1}\right) & \text { if } g \in K \\ 0 & \text { otherwise }\end{cases}
$$

It is not hard to check that this is an isomorphism of left $\mathscr{H}_{0}$-modules.

Composing with this isomorphism, the intertwining integral $A_{w}$ thus gives rise to a homomorphism $\mathscr{H}_{0} \rightarrow \mathscr{H}_{0}$. This can have poles (in the singular set) or zeros (if $\tau\left(a_{\alpha^{\vee}}\right)=q^{ \pm 1}$ for some coroot $\alpha^{\vee}$ ), but if $\tau$ is in general position, it is an isomorphism and so it agrees with right multiplication by a particular element $F_{w}$ of $\mathscr{H}_{0}$, which was identified by Rogawski [1985]. It is sufficient to describe it when $w=s_{i}$ is a simple reflection, and in this case

$$
F_{s_{i}}=\frac{1}{q}\left(T_{i}+1\right)-C_{\alpha_{i}}(\tau)
$$

where $C_{\alpha_{i}}(\tau)$ with $\tau=\tau_{z}$ is the ubiquitous rational function

$$
C_{\alpha_{i}}(\tau)=\frac{1-q^{-1} z^{\alpha_{i}^{\vee}}}{1-z^{\alpha_{i}^{\vee}}} .
$$


In other words, the following diagram is commutative:

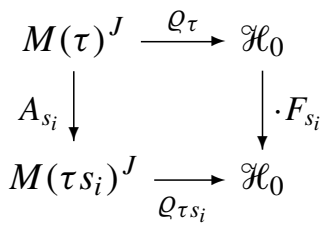

See [Brubaker, Bump and Licata 2011, Lemma 24] for a proof.

Instead of $\mathscr{H}_{0}$, one may also use $\mathscr{H}_{J}$ to model the intertwining integrals. The factor $C_{\alpha_{i}}(\tau)$, which depends on the spectral parameter, may then be replaced by an element of $\theta\left(P^{\vee}\right)$. This point of view is taken in Haines, Kottwitz and Prasad [Haines et al. 2010]. In order to see how this should work, consider that since the intertwining operator permutes the spaces $M(\tau)^{J}$, one might consider each $A_{w}$ to be an endomorphism of

$$
\bigoplus_{w \in W_{0}} M(\tau w)^{J}
$$

The characters $\tau$ of $B(F)$ that are induced all have a common trivial restriction to the subgroup $B_{0}=T(\mathfrak{o}) N(F)$, so by Frobenius reciprocity, the module $M=\operatorname{ind}_{B_{0}}^{G(F)}(1)$ is the direct integral of the spaces (5), with $\tau$ running over $\hat{T}$ modulo the action of $W_{0}$. This is the universal principal series. It is almost true that the intertwining integrals are endomorphisms of $\operatorname{ind}_{B_{0}}^{G(F)}(1)$; the difficulty is that the operators are polar in the singular set, so one must restrict to the orthogonal complement in $M$ of the spaces (5) with singular $\tau$. Alternatively, one may consider the compact induction $M_{c}=\mathrm{c}-i n d_{B_{0}}^{G(F)}(1)$. Although this is no longer closed under the intertwining operators, at least for $f$ in $M_{c}$ the intertwining integral (2) is always convergent, and $A_{w}$ is realized as a map $M_{c} \rightarrow M$.

We now come to the point, which is that as an $\mathscr{H}_{J}$-module, $M_{c}$ is a free module of rank one. This is to be expected from the Bruhat decomposition, because every coset in $B_{0} \backslash G(F) / J$ has a unique representative in the extended affine Weyl group, and this is also in bijection with $J \backslash G(F) / J$. Thus the extended affine Weyl group parametrizes both a basis of $M_{c}$ and a basis of $\mathcal{H}_{J}$. For a proof that the module $M_{c}$ is one-dimensional, see [Chriss and Khuri-Makdisi 1998] or [Haines et al. 2010, Lemma 1.6.1].

If $A_{w}$ were a map $M_{c} \rightarrow M_{c}$, we could then transfer $A_{w}$ to a map $\mathscr{H}_{J} \rightarrow \mathscr{H}_{J}$ and conclude that it agreed with right multiplication by some element. Due to the poles of the intertwining operators, this does not quite work. What may be done is to consider a somewhat larger Hecke algebra. In the Bernstein presentation $\mathscr{H}_{J} \cong \mathscr{H}_{0} \otimes \mathbb{C}\left[P^{\vee}\right]$, we can enlarge $\mathbb{C}\left[P^{\vee}\right]$ to any ring $\mathfrak{R}$ such that $\mathbb{C}\left[P^{\vee}\right] \subseteq \Re \subseteq \mathfrak{M}$, where $\mathfrak{M}$ is the field of fractions of $\mathbb{C}\left[P^{\vee}\right]$. We take $\mathfrak{R}$ to be the localization at the 
set of singular hyperplanes, that is, the ring obtained by adjoining $1-\alpha^{\vee}$ for all coroots $\alpha^{\vee}$. Let $\mathscr{H}_{J}^{\prime}=\mathscr{H}_{0} \otimes \Re$. Since the poles of the intertwining operators are contained in the singular locus, if $M^{\prime}$ denotes the submodule of $M$ generated by the image of the $A_{w}$, then we have a commutative diagram

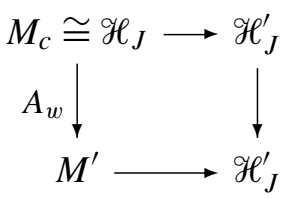

The top arrow is the natural inclusion and the bottom arrow extends the injection of $M_{c}$ into $\mathcal{H}_{J}$. The vertical arrows are $\mathscr{H}_{J}^{\prime}$-module homomorphisms. As before, if $R$ is a ring, then a left $R$-module homomorphism $R \rightarrow R$ is multiplication by some element, and therefore $A_{w}$ may be realized as multiplication by some element of $\mathcal{H}_{J}^{\prime}$.

\section{Hecke algebra modules from unique functionals}

There are two different kinds of actions of the affine Hecke algebra that we need to consider. First, in any smooth representation, the Hecke algebra acts by convolution. The Hecke algebra action on the Iwahori-fixed vectors in an induced representation is an action of this type.

Second, with the notation as in the prior section, the affine Hecke algebra acts on the ring $\mathcal{O}(\hat{T})$ of rational functions on the maximal torus $\hat{T}$ in the L-group. This ring is isomorphic to the group algebra $\mathbb{C}\left[P^{\vee}\right]$ of the coweight lattice $P^{\vee}$ of $T$, which may be identified with the weight lattice of $\hat{T}$. The generators $T_{i}$ of the finite Hecke algebra $\mathscr{H}_{0}$ will act by so-called Demazure-Lusztig operators. This action was introduced by Lusztig [1985] and has far-reaching consequences.

We have seen in the previous section how the Hecke algebra can be used to model intertwining integrals. In this section, we will show how we may translate this action of the Hecke algebra into an action on rational functions. We described two interpretations of intertwining operators via Hecke algebras in the diagrams (4) and (6); the simpler point of view in (4) will be sufficient for our purposes, as it was for Rogawski.

Let us consider, for every $z$ in some Zariski-dense subset of $\hat{T}$, a linear functional $L_{z}$ on $M(\tau)$. We shall suppose that $L_{z}$ arises from a multiplicity-free representation in the following way. Let $H$ be a subgroup of $G(F)$ and $\eta$ a character of $H$ such that the induced representation $\operatorname{ind}_{H}^{G(F)}(\eta)$ is multiplicity-free. Then by Frobenius reciprocity, a functional $L_{z}$ (if it exists) is characterized up to scalar multiple by the property that $L_{z}(\pi(g) \phi)=\eta(g) L_{z}(\phi)$ for $g \in H$. As noted above, using the uniqueness of the functional, it is often possible to show by a method of Bernstein that $L_{z}(\phi)$ is a rational function of $z$ on this set. Alternatively, this rationality may 
be proved for one specific vector, together with recursions that imply the rationality for all $\phi$.

As particular examples, $L_{z}$ could be the spherical functional

$$
\mathscr{S}_{z}(f)=\int_{K} f(k) d k
$$

or the Whittaker functional

$$
\mathscr{W}_{z}(f)=\int_{N(F)} f\left(w_{0} n g\right) \psi(n) d n,
$$

where $\psi$ is a nondegenerate character of $N(F)$, the unipotent radical of the Borel, and $w_{0}$ is the long element of the Weyl group $W$. The spherical and Whittaker functionals are both characterized up to scalar multiple by the uniqueness property described above. Indeed, since the spherical Hecke algebra of compactly supported $K$-biinvariant functions is commutative, $K$ is a Gelfand subgroup of $G(F)$, so the functional $L_{z}$ is determined (up to scalar) by the fact that $\mathscr{S}_{z}(\pi(k) \phi)=\mathscr{Y}_{z}(\phi)$ for all $k \in K$. For the Whittaker functional, the corresponding uniqueness result was obtained by Gelfand and Graev, by Piatetski-Shapiro and by Shalika [1974]. The Whittaker integral, like the intertwining integral, is convergent for $z$ in an open set, and has analytic continuation to all $z$.

It follows from these uniqueness results that for every pair $z$ and $w$, the functional $L_{z w} \circ A_{w}$ is a constant multiple of $L_{z}$. For the two examples above, these constants were computed by Casselman [1980] and Casselman and Shalika [1980], who found that for the spherical functional

$$
\mathscr{S}_{z w} \circ A_{w}=\prod_{\substack{\alpha \in \Delta^{+} \\ w^{-1} \alpha \in \Delta^{-}}}\left(\frac{1-q^{-1} z^{\alpha^{\vee}}}{1-z^{\alpha^{\vee}}}\right) \mathscr{S}_{z}=\prod_{\substack{\alpha \in \Delta^{+} \\ w^{-1} \alpha \in \Delta^{-}}} C_{\alpha}(\tau) \mathscr{S}_{z},
$$

with $C_{\alpha}(\tau)$ as in (3), and for the Whittaker functional

$$
W_{z w} \circ A_{w}=\prod_{\substack{\alpha \in \Delta^{+} \\ w^{-1} \alpha \in \Delta^{-}}}\left(\frac{1-q^{-1} z^{-\alpha^{\vee}}}{1-z^{\alpha^{\vee}}}\right) W_{z} .
$$

Here $\Delta^{+}$and $\Delta^{-}$are the positive and negative roots, and if $\alpha$ is a root, then $\alpha^{\vee}$ is the associated coroot.

Our goal is to describe a Hecke algebra action on $M(\tau)^{J}$ arising from each of these functionals and explain how this action gives a recursion for $L_{z}\left(\pi(g) \Phi_{w}\right)$ for any standard basis element $\Phi_{w} \in M(\tau)^{J}$. To describe the function $L_{z}\left(\pi(g) \Phi_{w}\right)$, it suffices to choose $g$ from a set of representatives for $H \backslash G(F) / J$, where $H=K$ when $L=\mathscr{Y}$ and $H=N$ when $L=\mathcal{W}$. This means that we may choose $g=a_{\lambda}$, where $\lambda \in P^{\vee}$, and in the Whittaker case we may assume $\lambda$ is dominant, since 
otherwise $\mathcal{W}\left(\pi\left(a_{\lambda}\right) \phi\right)=0$ for any $\phi \in M(\tau)$; see [Brubaker, Bump and Licata 2011, Lemma 5].

For both the spherical and Whittaker functionals, there is a standard basis vector $\Phi_{w}$ for which $L_{z}\left(\pi\left(a_{\lambda}\right) \Phi_{w}\right)$ has a particularly simple form. In the Whittaker case, for any dominant weight $\lambda$ we have

$$
\mathscr{W}_{z}\left(\pi\left(a_{\lambda}\right) \Phi_{w_{0}}^{\tau}\right)=\delta^{1 / 2}\left(a_{\lambda}\right) z^{w_{0} \lambda}
$$

see [Brubaker, Bump and Licata 2011, Proposition 6]. In the spherical case, we have: Proposition 1. Let $\lambda \in P^{\vee}$. Then $\mathscr{S}_{z}\left(\pi_{z}\left(a_{\lambda}\right) \Phi_{1}^{\tau}\right)=c(\lambda) z^{\lambda}$, where the constant $c(\lambda)$ is independent of $z$.

Proof. We have

$$
\mathscr{S}_{z}\left(\pi_{z}\left(a_{\lambda}\right) \Phi_{1}^{\tau}\right)=\int_{K} \Phi_{1}\left(k a_{\lambda}\right) d k=\left(\delta^{1 / 2} \tau\right)\left(a_{\lambda}\right) \int_{K} \Phi_{1}\left(a_{\lambda}^{-1} k a_{\lambda}\right) d k .
$$

The support of $\Phi_{1}$ is $B(F) J$. It is easy to see that if $k \in K$ and $a_{\lambda}^{-1} k a_{\lambda} \in B(F) J$, then $a_{\lambda}^{-1} k a_{\lambda} \in B_{0} J$, where $B_{0}$ is the kernel of $\tau_{z}: B(F) \rightarrow \mathbb{C}$. Hence the integral is a constant independent of $z$.

For either functional, this choice of standard basis vector will be the starting point for our recursion. It remains to define the Hecke action. From Rogawski's perspective, $M(\tau)^{J}$ is understood abstractly as a Hecke algebra module via the regular representation, which is determined by

$$
T_{w} \Phi_{1}=\Phi_{w} \quad \text { and } \quad T_{w} \Phi_{y}=T_{w} T_{y} \Phi_{1} \quad \text { for all } y, w \in W .
$$

Remembering the quadratic relation given in (1), we see that for a simple reflection $s$,

$$
T_{s} \Phi_{w}= \begin{cases}\Phi_{s w} & \text { if } s w>w \\ q \Phi_{s w}+(q-1) \Phi_{w} & \text { if } s w<w .\end{cases}
$$

But (4) gives a relation between the intertwining operator $A_{s}$ and $T_{s}$. So combining these two ingredients and manipulating the terms, we obtain the following result.

Proposition 2. Let $\tau=\tau_{z}$ and let $w \in W_{0}$. Let $s=s_{i}$ be a simple reflection. Then

$$
A_{s} \Phi_{w}^{\tau s}+C_{\alpha}(\tau) \Phi_{w}^{\tau}= \begin{cases}\Phi_{w}^{\tau}+\Phi_{s w}^{\tau} & \text { if } s w<w \\ q^{-1}\left(\Phi_{w}^{\tau}+\Phi_{s w}^{\tau}\right) & \text { if } s w>w .\end{cases}
$$

Proof. This is an easy consequence of [Casselman 1980, Theorem 3.4]; see Proposition 8 of [Brubaker, Bump and Licata 2011].

Thus it is reasonable to expect that we may obtain an action of the Hecke algebra on the ring $\mathcal{O}(\hat{T})$ of regular functions on $\hat{T}$ via the regular representation by applying the functional to both sides of (9). 
The case where $L=\mathscr{W}$ is treated in detail in [Brubaker, Bump and Licata 2011], so we will focus on the case $L=\mathscr{Y}$, and return at the end to make some remarks about the difference between the two cases. For $L=\mathscr{Y}$, we intend to start our recursion at $\Phi_{1}$ and move up in the Bruhat order. So let us rewrite (9) in the case $s w>w$ :

$$
q A_{s} \Phi_{w}^{\tau s}+\left(q C_{\alpha}(\tau)-1\right) \Phi_{w}^{\tau}=\Phi_{s w}^{\tau} .
$$

We will find that applying $\mathscr{S}_{z}$ to both sides gives a recursive identity for the matrix coefficient

$$
F_{\lambda, w}(z):=\mathscr{Y}_{z}\left(\pi\left(a_{\lambda}\right) \Phi_{w}^{\tau}\right)
$$

of the principal series representation. To state this more precisely, let us introduce the Demazure-Lusztig operator $\mathscr{T}_{i}$ defined on an arbitrary function $F$ on the dual torus $\hat{T}$ by

$$
\mathscr{T}_{i} F(z):=q C_{-\alpha_{i}}(\tau) F\left(z s_{i}\right)+\left(q C_{\alpha_{i}}(\tau)-1\right) F(z) .
$$

Here $1 \leq i \leq r$ corresponds to a simple reflection $s_{i}$ of the finite Weyl group. After some algebra, this is equivalent to

$$
\mathscr{T}_{i} F(z)=\left(z^{\alpha_{i}}-1\right)^{-1}\left(F(z)-F\left(z s_{i}\right)-q F(z)+q z^{\alpha_{i}} F\left(z s_{i}\right)\right),
$$

which is precisely the operator defined in [Lusztig 1985, (8.1)]. (This can also be defined if $i=0$, but for this discussion we are excluding this case.)

The following result is equivalent to a result of Ion [2006, Proposition 5.8].

Theorem 1. The Demazure-Lusztig operators satisfy the quadratic and braid relations, and hence generate a ring isomorphic to the Hecke algebra $\mathscr{H}_{0}$. If $w \in W_{0}$ and $s_{i} w>w$, then

$$
F_{\lambda, s_{i} w}=\mathscr{T}_{i} F_{\lambda, w} .
$$

The fact that the Demazure-Lusztig operators satisfy the quadratic and braid relations is due to Lusztig [1985, Section 8]. However, checking the braid relations directly depends on a tedious computation for rank-2 root systems, so it may be of interest that we can avoid such computations using our methods.

Proof. Assume that $s w>w$. Apply $\pi\left(a_{\lambda}\right)$ to both sides of (10) and then apply $\mathscr{Y}_{z}$. We obtain

$$
F_{\lambda, s w}(z)=q_{z}^{\tau} A_{s}\left(\pi\left(a_{\lambda}\right) \Phi_{w}^{\tau s}\right)+\left(q C_{\alpha}(\tau)-1\right) F_{\lambda, w}(z) .
$$

Now we use (7), replacing $z$ by $z s$ and remembering that $C_{\alpha}\left(\tau_{z s}\right)=C_{-\alpha}(\tau)$. Comparing with (11), the right-hand side of (13) is just $\mathscr{T}_{i} F_{\lambda, w}^{\tau}$, as desired.

We turn to the fact that the $\mathscr{T}_{i}$ satisfy the generating relations of $\mathscr{H}_{0}$. Let $m$ be the order of $s_{i} s_{j}$ with $1 \leqslant i, j \leqslant r, i \neq j$. To show that $\mathscr{T}_{i} \mathscr{T}_{j} \mathscr{T}_{i} \ldots=\mathscr{T}_{j} \mathscr{T}_{i} \mathscr{T}_{j} \ldots$ 
( $m$ factors on both sides), it is sufficient to show that they have the same effect on $z^{\lambda}$, where $\lambda \in P^{\vee}$. By Proposition 1, it is thus sufficient to show that

$$
\mathscr{T}_{i} \mathscr{T}_{j} \mathscr{T}_{i} \ldots F_{a_{\lambda}, 1}(z)=\mathscr{T}_{j} \mathscr{T}_{i} \mathscr{T}_{j} \ldots F_{a_{\lambda}, 1}(z)
$$

But applying (12), both sides equal $F_{a_{\lambda}, w}(z)$, where $w=s_{i} s_{j} s_{i} \ldots=s_{j} s_{i} s_{j} \ldots$ is the longest element of the dihedral group generated by $s_{i}$ and $s_{j}$.

We next prove the quadratic relation. Assume now that $s_{i} w<w$. Then applying (12) to $F_{\lambda, s_{i} w}$, we have $F_{\lambda, s_{i} w}=\mathscr{T}_{i}^{-1} F_{\lambda, w}$. Now we can compute this by the first case of (9), and we find

$$
\mathscr{T}_{i}^{-1} F(z)=C_{-\alpha} F\left(z s_{i}\right)+\left(C_{\alpha}(\tau)-1\right) F(z) .
$$

This means that $\mathscr{T}_{i}^{-1}=q^{-1} \mathscr{T}_{i}+q^{-1}-1$, which is equivalent to the quadratic relation $\mathscr{T}_{i}^{2}=(q-1) \mathscr{T}_{i}+q$.

This theorem guarantees that, given any reduced decomposition for the Weyl group element $w=s_{i_{1}} \ldots s_{i_{k}}$, the operator

$$
\mathscr{T}_{w}:=T_{i_{1}} \ldots \mathscr{T}_{i_{k}}
$$

is well defined. As noted in [Ion 2008, Theorem 3.1], the Demazure-Lusztig operators applied to $z^{\lambda}$ for $\lambda$ dominant give a recursive definition for a certain limit of nonsymmetric Macdonald polynomials with weight $w \cdot \lambda \in P^{\vee}$. Thus our Iwahori-spherical functions are also limits of these polynomials for $\lambda$ dominant.

We caution the reader that our conventions for Demazure-Lusztig operators and nonsymmetric Macdonald polynomials differ slightly from those of Ion. Instead, they more closely parallel those of Cherednik [1995]. In particular, our $\mathscr{T}_{i}$ essentially match those in [Cherednik 1995, (3.5)], which are then used to construct nonsymmetric Macdonald polynomials.

Returning to the Whittaker case, similar arguments to those presented above were given in [Brubaker, Bump and Licata 2011]. The resulting operators are not Demazure-Lusztig operators, but are related in a way that is made precise in Section 5 of that reference. The difference results from the fact that the starting point for the Whittaker recursion is the Iwahori-fixed vector $\Phi_{w_{0}}$, rather than $\Phi_{1}$. Furthermore, the constant of proportionality in (8) differs slightly from the spherical case given in (7). The resulting recursive operators for Whittaker functions are Demazure-Lusztig operators conjugated by $\theta\left(\rho^{\vee}\right)$ and with $q$ replaced by $q^{-1}$. Here $\theta: \mathbb{C}\left[P^{\vee}\right] \rightarrow \mathscr{H}_{J}$ is as in the previous section and $\rho^{\vee}$ is half the sum of the positive coroots. In either the spherical or Whittaker cases, the resulting action of the finite Hecke algebra can be generalized to an action of the (extended) affine Hecke algebra where the elements in $\theta\left(P^{\vee}\right)$ act by translation. (See Theorem 28 of [Brubaker, Bump and Licata 2011] for the Whittaker case.) 


\section{Acknowledgements}

We thank David Kazhdan for helpful conversations regarding universal principal series and Daniel Orr for discussions on nonsymmetric Macdonald polynomials.

\section{References}

[Banks 1998] W. D. Banks, "A corollary to Bernstein's theorem and Whittaker functionals on the metaplectic group”, Math. Res. Lett. 5:6 (1998), 781-790. MR 99m:11059 Zbl 0944.22007

[Bourbaki 1968] N. Bourbaki, Eléments de mathématique: groupes et algèbres de Lie, Hermann, Paris, 1968. Translated by Pressley, A., Springer, Berlin, 2002. MR 2003a:17001 Zbl 0983.17001

[Brubaker, Bump and Licata 2011] B. Brubaker, D. Bump, and A. Licata, "Whittaker functions and Demazure operators", preprint, 2011. arXiv 1111.4230

[Casselman 1980] W. Casselman, "The unramified principal series of $\mathfrak{p}$-adic groups, I: The spherical function”, Compositio Math. 40:3 (1980), 387-406. MR 83a:22018 Zbl 0472.22004

[Casselman and Shalika 1980] W. Casselman and J. Shalika, "The unramified principal series of p-adic groups, II: The Whittaker function”, Compositio Math. 41:2 (1980), 207-231. MR 83i:22027 Zbl 0472.22005

[Cherednik 1995] I. Cherednik, "Nonsymmetric Macdonald polynomials", Internat. Math. Res. Notices 10 (1995), 483-515. MR 97f:33032 Zbl 0886.05121

[Cherednik and Ostrik 2003] I. Cherednik and V. Ostrik, "From double Hecke algebra to Fourier transform”, Selecta Math. (N.S.) 9:2 (2003), 161-249. MR 2004f:20011 Zbl 1027.22014

[Chriss and Khuri-Makdisi 1998] N. Chriss and K. Khuri-Makdisi, "On the Iwahori-Hecke algebra of a p-adic group”, Internat. Math. Res. Notices 2 (1998), 85-100. MR 99c:22023 Zbl 0894.22013

[Haines et al. 2010] T. J. Haines, R. E. Kottwitz, and A. Prasad, "Iwahori-Hecke algebras", J. Ramanujan Math. Soc. 25:2 (2010), 113-145. MR 2011e:22022 Zbl 1202.22013

[Ion 2006] B. Ion, "Nonsymmetric Macdonald polynomials and matrix coefficients for unramified principal series”, Adv. Math. 201:1 (2006), 36-62. MR 2006k:22016 Zbl 1103.33010

[Ion 2008] B. Ion, "Standard bases for affine parabolic modules and nonsymmetric Macdonald polynomials", J. Algebra 319:8 (2008), 3480-3517. MR 2010c:20004 Zbl 1150.22005

[Iwahori and Matsumoto 1965] N. Iwahori and H. Matsumoto, "On some Bruhat decomposition and the structure of the Hecke rings of p-adic Chevalley groups", Inst. Hautes Études Sci. Publ. Math. 25 (1965), 5-48. MR 32 \#2486 Zbl 0228.20015

[Jacquet and Rallis 1996] H. Jacquet and S. Rallis, "Uniqueness of linear periods", Compositio Math. 102:1 (1996), 65-123. MR 97k:22025 Zbl 0855.22018

[Jantzen 1979] J. C. Jantzen, Moduln mit einem höchsten Gewicht, Lecture Notes in Mathematics 750, Springer, Berlin, 1979. MR 81m:17011 Zbl 0426.17001

[Kazhdan and Lusztig 1979] D. Kazhdan and G. Lusztig, "Representations of Coxeter groups and Hecke algebras”, Invent. Math. 53:2 (1979), 165-184. MR 81j:20066 Zbl 0499.20035

[Kazhdan and Lusztig 1985] D. Kazhdan and G. Lusztig, "Equivariant $K$-theory and representations of Hecke algebras, II”, Invent. Math. 80:2 (1985), 209-231. MR 88f:22054b Zbl 0613.22003

[Kazhdan and Lusztig 1987] D. Kazhdan and G. Lusztig, "Proof of the Deligne-Langlands conjecture for Hecke algebras", Invent. Math. 87:1 (1987), 153-215. MR 88d:11121 Zbl 0613.22004

[Lusztig 1985] G. Lusztig, "Equivariant $K$-theory and representations of Hecke algebras", Proc. Amer. Math. Soc. 94:2 (1985), 337-342. MR 88f:22054a Zbl 0571.22014 
[Lusztig 1989] G. Lusztig, "Affine Hecke algebras and their graded version”, J. Amer. Math. Soc. 2:3 (1989), 599-635. MR 90e:16049 Zbl 0715.22020

[Macdonald 2003] I. G. Macdonald, Affine Hecke algebras and orthogonal polynomials, Cambridge Tracts in Mathematics 157, Cambridge University Press, Cambridge, 2003. MR 2005b:33021 Zbl 1024.33001

[Opdam 1995] E. M. Opdam, "Harmonic analysis for certain representations of graded Hecke algebras”, Acta Math. 175:1 (1995), 75-121. MR 98f:33025 Zbl 0836.43017

[Reeder 1992] M. Reeder, "On certain Iwahori invariants in the unramified principal series", Pacific J. Math. 153:2 (1992), 313-342. MR 93b:22033 Zbl 0804.22010

[Reeder 1993] M. Reeder, "p-adic Whittaker functions and vector bundles on flag manifolds", Compositio Math. 85:1 (1993), 9-36. MR 93m:22020 Zbl 0819.22012

[Rogawski 1985] J. D. Rogawski, “On modules over the Hecke algebra of a p-adic group”, Invent. Math. 79:3 (1985), 443-465. MR 86j:22028 Zbl 0579.20037

[Sakellaridis 2006] Y. Sakellaridis, "A Casselman-Shalika formula for the Shalika model of GL ${ }_{n}$ ", Canad. J. Math. 58:5 (2006), 1095-1120. MR 2007m:22017 Zbl 1114.22011

[Shalika 1974] J. A. Shalika, "The multiplicity one theorem for GL ${ }_{n}$ ", Ann. of Math. (2) 100 (1974), 171-193. MR 50 \#545 Zbl 0316.12010

[Zelevinsky 1980] A. V. Zelevinsky, "Induced representations of reductive p-adic groups, II: On irreducible representations of GL(n)", Ann. Sci. École Norm. Sup. (4) 13:2 (1980), 165-210. MR 83g:22012 Zbl 0441.22014

[Zelevinsky 1981] A. V. Zelevinsky, "The p-adic analogue of the Kazhdan-Lusztig conjecture", Funktsional. Anal. i Prilozhen. 15:2 (1981), 9-21. In Russian; translated in Funct. Anal. Appl. 15:2 (1981), 83-92. MR 84g:22039 Zbl 0463.22013

Received September 13, 2012.

BENJAMIN BRUBAKER

SCHOOL OF MATHEMATICS

UNIVERSITY OF MINNESOTA

MINNEAPOLIS, MN 55455

UNITED STATES

brubaker@math.umn.edu

DANIEL BUMP

DEPARTMENT OF MATHEMATICS

STANFORD UNIVERSITY

BUILDING 380

STANFORD, CA 94305-2125

UNITED STATES

bump@math.stanford.edu

SOLOMON FRIEDBERG

DEPARTMENT OF MATHEMATICS

BOSTON COLLEGE

Chestnut Hill MA 02467-3806

UNITED STATES

friedber@bc.edu 


\title{
PACIFIC JOURNAL OF MATHEMATICS
}

\author{
http://pacificmath.org
}

Founded in 1951 by E. F. Beckenbach (1906-1982) and F. Wolf (1904-1989)

\section{EDITORS}

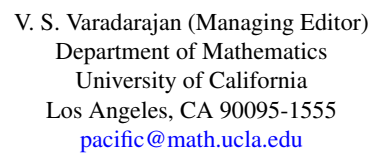

Don Blasius

Department of Mathematics University of California

Los Angeles, CA 90095-1555

blasius@math.ucla.edu

Robert Finn

Department of Mathematics

Stanford University

Stanford, CA 94305-2125

finn@math.stanford.edu

Alexander Merkurjev

Department of Mathematics

University of California

Los Angeles, CA 90095-1555

merkurev@math.ucla.edu

\author{
Vyjayanthi Chari \\ Department of Mathematics \\ University of California \\ Riverside, CA 92521-0135 \\ chari@math.ucr.edu \\ Kefeng Liu \\ Department of Mathematics \\ University of California \\ Los Angeles, CA 90095-1555 \\ liu@math.ucla.edu \\ Sorin Popa \\ Department of Mathematics \\ University of California \\ Los Angeles, CA 90095-1555 \\ popa@math.ucla.edu \\ Paul Yang \\ Department of Mathematics \\ Princeton University \\ Princeton NJ 08544-1000 \\ yang@math.princeton.edu
}

\section{PRODUCTION}

Silvio Levy, Scientific Editor, pacific@math.berkeley.edu

\section{SUPPORTING INSTITUTIONS}

ACADEMIA SINICA, TAIPEI

CALIFORNIA INST. OF TECHNOLOGY

INST. DE MATEMÁTICA PURA E APLICADA

KEIO UNIVERSITY

MATH. SCIENCES RESEARCH INSTITUTE

NEW MEXICO STATE UNIV.

OREGON STATE UNIV.

\author{
STANFORD UNIVERSITY \\ UNIV. OF BRITISH COLUMBIA \\ UNIV. OF CALIFORNIA, BERKELEY \\ UNIV. OF CALIFORNIA, DAVIS \\ UNIV. OF CALIFORNIA, LOS ANGELES \\ UNIV. OF CALIFORNIA, RIVERSIDE \\ UNIV. OF CALIFORNIA, SAN DIEGO \\ UNIV. OF CALIF., SANTA BARBARA
}

\author{
Daryl Cooper \\ Department of Mathematics \\ University of California \\ Santa Barbara, CA 93106-3080 \\ cooper@math.ucsb.edu \\ Jiang-Hua Lu \\ Department of Mathematics \\ The University of Hong Kong \\ Pokfulam Rd., Hong Kong \\ jhlu@maths.hku.hk
}

\section{Jie Qing}

Department of Mathematics

University of California

Santa Cruz, CA 95064

qing@cats.ucsc.edu

These supporting institutions contribute to the cost of publication of this Journal, but they are not owners or publishers and have no responsibility for its contents or policies.

See inside back cover or pacificmath.org for submission instructions.

The subscription price for 2012 is US \$420/year for the electronic version, and \$485/year for print and electronic.

Subscriptions, requests for back issues from the last three years and changes of subscribers address should be sent to Pacific Journal of Mathematics, P.O. Box 4163, Berkeley, CA 94704-0163, U.S.A. Prior back issues are obtainable from Periodicals Service Company, 11 Main Street, Germantown, NY 12526-5635. The Pacific Journal of Mathematics is indexed by Mathematical Reviews, Zentralblatt MATH, PASCAL CNRS Index, Referativnyi Zhurnal, Current Mathematical Publications and the Science Citation Index.

The Pacific Journal of Mathematics (ISSN 0030-8730) at the University of California, c/o Department of Mathematics, 969 Evans Hall, Berkeley, CA 94720-3840, is published monthly except July and August. Periodical rate postage paid at Berkeley, CA 94704, and additional mailing offices. POSTMASTER: send address changes to Pacific Journal of Mathematics, P.O. Box 4163, Berkeley, CA 94704-0163.

PJM peer review and production are managed by EditFlow ${ }^{\circledR}$ from Mathematical Sciences Publishers.

\section{PUBLISHED BY}

\section{mathematical sciences publishers}

http://msp.org/

A NON-PROFIT CORPORATION

Typeset in LATEX 


\section{PACIFIC JOURNAL OF MATHEMATICS}

Volume $260 \quad$ No. $2 \quad$ December 2012

\section{Special issue \\ devoted to the memory of Jonathan Rogawski}

In memoriam: Jonathan Rogawski

257

DON BLASIUS, DINAKAR RAMAKRISHNAN and V. S. VARADARAJAN

$p$-adic Rankin $L$-series and rational points on CM elliptic curves

261

Massimo Bertolini, Henri DARMON and KARTIK PRASANNA

The syntomic regulator for $K_{4}$ of curves

AMNON BESSER and ROB DE JEU

Unique functionals and representations of Hecke algebras

381

BENJAMIN BRUBAKER, DANIEL BUMP and SOLOMON FRIEDBERG

A relative trace formula for PGL(2) in the local setting

395

BROOKE FEIGON

On the degrees of matrix coefficients of intertwining operators

433

TOBIAS FINIS, EREZ LAPID and WERNER MÜLlER

Comparison of compact induction with parabolic induction

457

Guy HENNIART and MARIE-FranCE Vigneras

The functional equation and beyond endoscopy

497

P. EDWARD HERMAN

A correction to Conducteur des Représentations du groupe linéaire

HERVÉ JACQUET

Modular $L$-values of cubic level

ANDREW KNIGHTLY and CHARLES LI

On occult period maps

STEPHEN KUDLA and MiCHAEL RAPOPORT

A prologue to "Functoriality and reciprocity", part I

ROBERT LANGLANDS

Truncation of Eisenstein series

EREZ LAPID and KeITH OUELLETTE

Some comments on Weyl's complete reducibility theorem

JONATHAN ROGAWSKI and V. S. VARADARAJAN

On equality of arithmetic and analytic factors through local Langlands correspondence

FREYDOON SHAHIDI 